\title{
Nanocomposites base polymère, renforcés par des particules rigides
}

\author{
Emmanuelle Chabert ${ }^{1,2}$, Laurent Chazeau ${ }^{1}$, Catherine Gauthier ${ }^{1}$, Rémy Dendievel ${ }^{3}$ \\ ET JeAn-Yves CAVAILLÉ ${ }^{1, a}$ \\ 1 GEMPPM, UMR INSA-CNRS, 5510, 69621 Villeurbanne Cedex, France \\ 2 LMS, UMR 7649, École polytechnique, 91128 Palaiseau Cedex, France \\ 3 GPM2, UMR INPG-CNRS, BP 46, 38402 Saint Martin d'Hères, France
}

Reçu le 10 juin 2003, accepté le 7 novembre 2003

\begin{abstract}
Résumé - Les matériaux composites à base polymère montrent, dans certains cas, des comportements mécaniques spécifiques lorsque la taille des renforts particulaires rigides devient nanométrique. Il semble que cette particularité provienne du fait que les distances inter-particulaires moyennes sont alors comparables aux dimensions caractéristiques des chaînes polymères. On observe parfois des effets de renfort beaucoup plus importants que pour des composites classiques qui peuvent être attribués à l'existence de réseaux particule/macromolécule/particule ou encore de réseaux rigides de particules. Dans cette courte revue, l'effet du facteur de forme, de la fraction volumique et du procédé de mise en oeuvre sont discutés, ainsi que l'état de l'art en ce qui concerne la modélisation dans les domaines linéaire et non linéaire.
\end{abstract}

Mots clés : Nanocomposites / polymères / percolation / propriétés mécaniques / modélisation

Abstract - Polymeric nanocomposites reinforced by stiff particles. Polymer based composite materials, often exhibit specific mechanical behaviors when the size of particulate fillers is in the nanometric range. It seems that this specificity could be related to the fact that in this case, the average distance between the filler surfaces has the same order of magnitude than the characteristic macromolecules dimensions, such as the coil diameter, etc. Above the glass transition temperature $T g$, these materials display sometimes a much larger reinforcement effect than for classical composites. This can be related to the existence of networks involving either particle/macromolecules/particle or direct particle/particle interactions. In this brief review, the effect of (i) filler shape factor and volume fraction, and (ii) the processing methods are discussed through several examples, as well as some information about modeling aspects, both under linear and non linear conditions.

Key words: Nanocomposites / polymers / fillers / percolation / mechanical properties / modeling

\section{Introduction}

La démarche qui consiste à associer divers matériaux entre eux, afin d'obtenir, en particulier, de meilleures propriétés mécaniques est extrêmement ancienne et est utilisée aussi par certaines espèces animales et de manière plus générale par la nature elle-même [1]. Le choix des matériaux dans une telle association dépend naturellement des propriétés visées. S'il s'agit de limiter la fragilité des polymères à l'état vitreux, comme pour le polystyrène (PS) par exemple, on introduit dans la matrice

a Auteur correspondant :

jean-yves.cavaille@insa-lyon.fr des nodules d'élastomère (par exemple polybutadiène), très souple aux températures d'utilisation du matériau. Cette association rend le PS résistant à l'impact (High Impact PS, HIPS). Si au contraire, on s'intéresse à une augmentation de la rigidité, on introduira des particules (beaucoup) plus rigides que la matrice. C'est typiquement le cas des composites à fibre de verre ou de carbone, c'est aussi le cas des caoutchoucs employés pour la fabrication des pneumatiques pour véhicules. On utilise alors des particules de taille nanométrique (noir de carbone ou silice).

S'il est bien connu que les propriétés macroscopiques des composites dépendent de la nature des constituants et de leur fraction volumique, de leur arrangement spatial et des propriétés interfaciales entre matrice et renforts, 
il était moins usuel, jusqu'à ces dernières années, de s'intéresser aux effets de taille et, en l'occurrence, aux cas où les dimensions caractéristiques deviennent nanométriques. Dans le cas des polymères, les distances inter-particules deviennent de l'ordre de grandeur du rayon de giration des macromolécules (soit quelques $\mathrm{nm}$ ), et si on admet l'existence d'une interphase où la mobilité des chaînes macromoléculaires est affectée par la présence des surfaces rigides (adsorption, interactions spécifiques, etc.), une fraction importante de matrice peut être concernée. Par ailleurs, les effets d'interaction particule-particule sont généralement ignorés pour les systèmes classiques, mais semblent pouvoir jouer un rôle prépondérant aux échelles nanoscopiques. La réponse de ces matériaux à une sollicitation mécanique (linéaire ou non) nécessite de prendre en compte ces effets pour l'analyse de leur comportement.

Nous allons passer en revue quelques aspects, liés à la dispersion des charges renforçantes, au rôle de leurs interactions et à l'analyse du comportement mécanique (linéaire et non linéaire). Pour avoir une information plus complète, le lecteur peut consulter la référence [2].

\section{Dispersion et percolation de particules renforçantes dans une matrice polymère}

Pour déterminer le rôle du type de dispersion (régulière, aléatoire) sur le comportement macroscopique, il faut naturellement disposer de matériaux spécifiques. Pour cette raison particulière, nous avons choisi de mettre en œuvre des matériaux composites à partir de procédés de polymérisation en émulsion, qui conduisent à des suspensions aqueuses colloïdales de particules polymères (latex), ayant des diamètres typiques de l'ordre de $100 \mathrm{~nm}$. L'intérêt du procédé est double. D'une part, il est facile de mélanger plusieurs suspensions colloïdales (encore faut-il veiller à la stabilité du mélange), et la filmification (évaporation de l'eau concomitante à la coalescence des particules les plus «molles ») produit directement des matériaux nano-hétérogènes. D'autre part, il est possible de polymériser différents monomères en plusieurs étapes, ce qui permet d'obtenir, si le procédé est bien contrôlé, des particules structurées. Ces particules peuvent être de type cour-couronne (core-shell) [3] ou encore contenir des particules inorganiques encapsulées (comme la silice $)^{1}$.

Le schéma suivant (Fig. 1) montre comment il est possible d'obtenir, après filmification, deux types de dispersions différentes, soit aléatoire (a) soit régulière (b). Dans le cas d'une dispersion régulière, on peut en principe atteindre des fractions volumiques de phases rigides proches de $50 \%$ sans contact entre les particules. Dans le cas de dispersions réellement aléatoires, on s'attend à ce que la topologie suive les lois de la percolation.

De tels nanocomposites constitués d'une matrice molle de polyacrylate de butyle ou $\mathrm{P}(\mathrm{ABu})$, dans laquelle des particules rigides de polystyrène (PS) sont dispersées soit

\footnotetext{
1 Travaux en collaboration avec E. Bourgeat Lami, LCPP, UMR 140 CPE, Lyon.
}

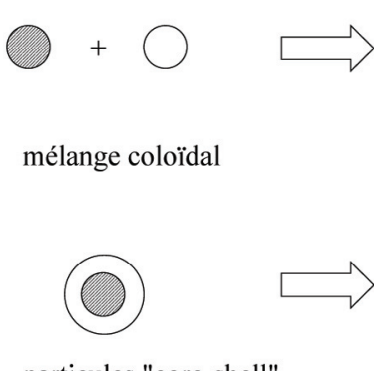

particules "core-shell"

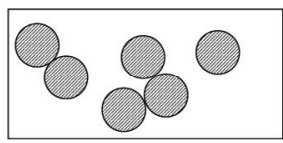

distribution aléatoire

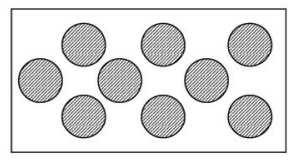

renforts sans contacts
Fig. 1. Obtention de matériaux modèles par deux voies modifiant la dispersion des particules renforçantes (grisées) dans la matrice (blanche).

aléatoirement soit régulièrement, ont été préparés pour $40 \%$ de phase rigide. La figure 2 montre l'évolution de leur module de cisaillement avec la température, mesurée après coalescence de la matrice $\mathrm{P}(\mathrm{ABu})$ (i) et après un traitement thermique au-dessus de la température de transition vitreuse du PS (j) [4]. Ces mesures ont été effectuées à l'aide d'un spectromètre mécanique travaillant en torsion à la fréquence constante de $1 \mathrm{~Hz}$, sous déformation relative maximum de $10^{-4}$. Juste après filmification, ces matériaux présentent des comportements légèrement différents. Après traitement thermique audessus de la température de transition vitreuse $(T g)$ du PS, le système «aléatoire » est notablement plus rigide que le système « régulier ». En fait, le matériau « aléatoire » est constitué d'un réseau percolant de particules sphériques rigides en contact dans une matrice continue (souple dans l'intervalle [220, $370 \mathrm{~K}]$ ).

$\mathrm{Au}$ cours du traitement thermique (qui autorise la diffusion des chaînes PS), ce réseau percolant peut coalescer, ce qui conduit, après retour à température ambiante, à la formation d'un réseau rigide. Ce phénomène n'est pas possible dans le deuxième système, puisque pour l'essentiel, les particules de PS ne sont pas en contact. Ces résultats expérimentaux illustrent la différence entre un état de percolation géométrique (surface de contact faible, transmission des forces normales et tangentielles entre particules de renfort) à un état de percolation mécanique (grande surface de contact, transmission des forces et des moments) [5].

Si on suppose que le phénomène de percolation est essentiel, alors deux paramètres sont à considérer : d'une part le facteur de forme $(\xi)$, défini comme le rapport entre la plus grande et la petite dimension, et d'autre part la distribution en orientation (très dépendante du procédé de mise en œuvre des composites). L'influence de ces deux paramètres sur le seuil de percolation est illustré sur la figure $3[6,7]$. Le seuil déterminé ici par simulation numérique correspond à la fraction volumique de phase rigide pour laquelle un premier chemin macroscopique apparaît. On peut remarquer la chute rapide du seuil de percolation lorsque le facteur de forme augmente (Fig. 3d). Une restriction des angles d'orientation des renforts conduit à l'augmentation de ce même seuil (Fig. 3e). 


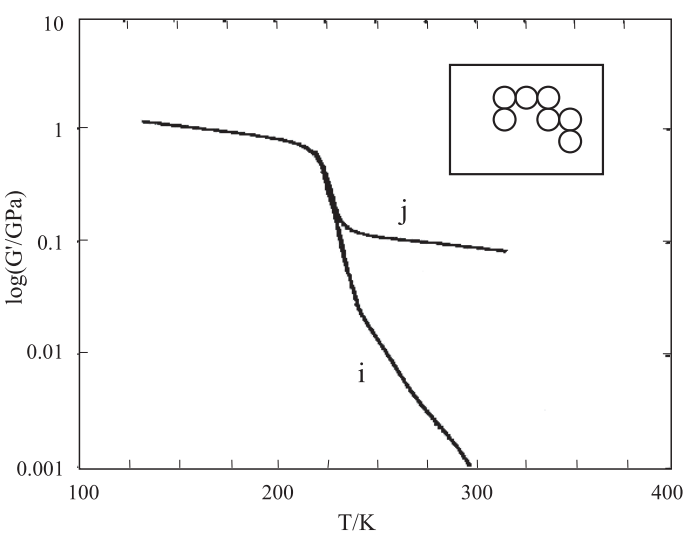

$\mathrm{a}$

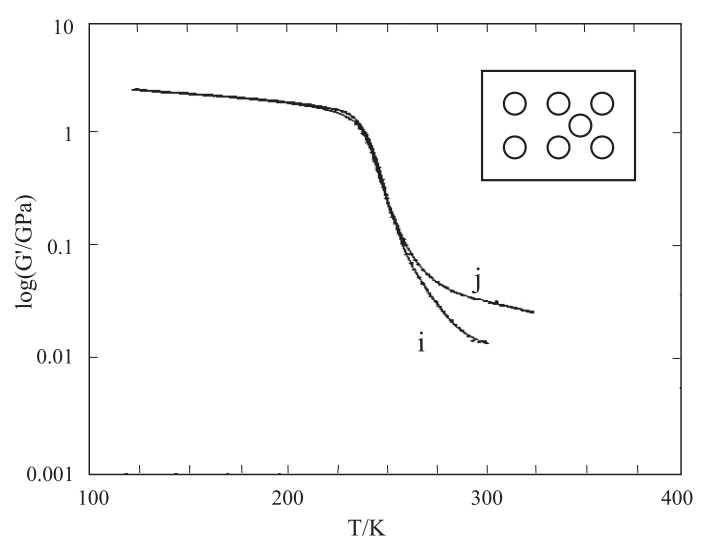

$\mathrm{b}$

Fig. 2. Comportement mécanique dynamique dans le cas où la phase rigide est constituée de particules polystyrène (PS) $\left(T g \approx 100{ }^{\circ} \mathrm{C}\right)$ et la phase souple de $\mathrm{p}(\mathrm{ABu})\left(T g \approx-50{ }^{\circ} \mathrm{C}\right)$. À gauche, le matériau est obtenu à partir du mélange des deux latex, à droite à partir de particules core-shell; (i) matériau après coalescence à $T_{a m b}$; (j) après traitement thermique à $140{ }^{\circ} \mathrm{C}$. Les fractions volumiques des constituants sont identiques dans les deux cas.

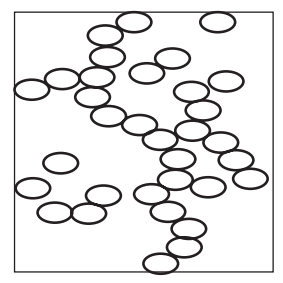

a

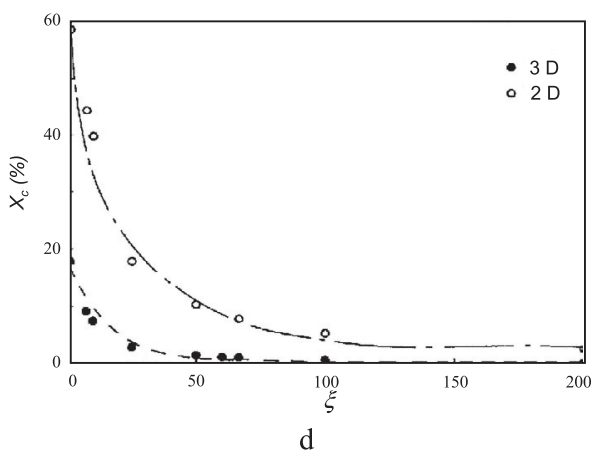

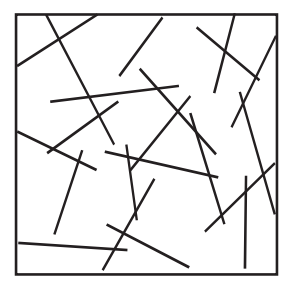

b

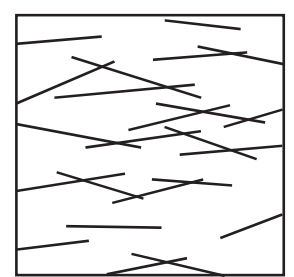

C

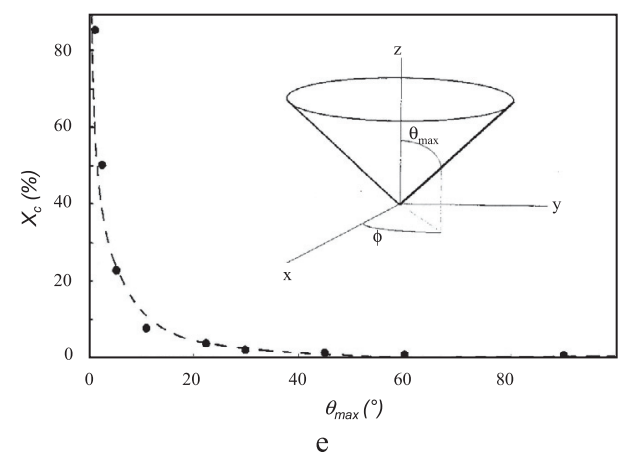

Fig. 3. Seuils de percolation en $2 \mathrm{D}$ et $3 \mathrm{D}$ en fonction de la forme des particules renforçantes (a) et (b) et de leur orientation (c). Pour des particules sphériques, ce seuil vaut $\approx 20 \%$. Pour des whiskers de facteur de forme de 100 , il vaut $1 \%$. Pour des whiskers tous parallèles, il atteint presque $100 \%$ [6].

On s'attend donc à des effets de renforcement marqués dans le cas de renforts à facteur de forme élevé lorsque ceux-ci peuvent former des liaisons rigides.

S'il est clair qu'un réseau rigide percolant renforce plus que des particules dispersées sans contact, il est important de mettre en relation la nature des interactions et l'effet de renfort.

\section{Rôle de la nature des interactions entre particules renforçantes}

L'exemple suivant porte sur des matériaux obtenus toujours par voie aqueuse, mais les charges renforçantes sont des monocristaux de cellulose (whiskers rigides, d'environ $10 \mathrm{~nm}$ de diamètre et $1 \mu \mathrm{m}$ de long). Deux procédés d'élaboration ont été utilisés. Le premier procédé consiste à mélanger une suspension colloïdale de whiskers avec un latex $\mathrm{p}(\mathrm{S}-\mathrm{ABu})$ et à réaliser les films composites par évaporation du mélange (matériaux E). Le second procédé consiste à lyophiliser le mélange (plutôt qu'à l'évaporer). Le lyophilisat ainsi obtenu est ensuite extrudé (matériaux XP).

La figure suivante (Fig. 4) présente les mesures de spectrométrie mécanique réalisées sur le système évaporé (E). Au-dessus de $T g$ de la matrice, l'effet de renfort est extrêmement élevé, puisque l'ajout de $6 \%$ de whiskers augmente le module d'un facteur 500 environ [8]. 


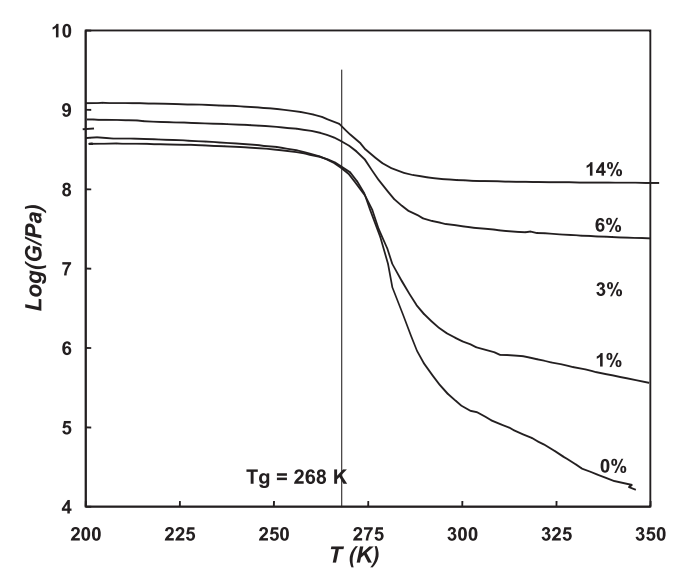

a

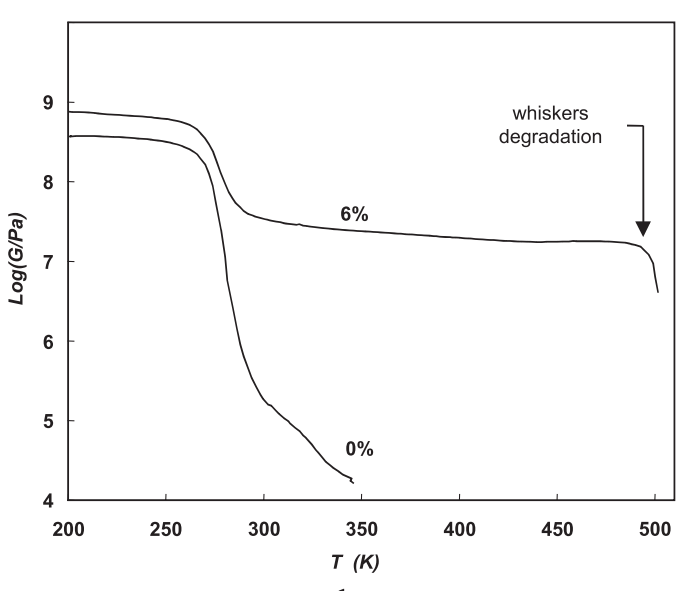

b

Fig. 4. Comportement mécanique dynamique de nanocomposites à matrice $\mathrm{P}(\mathrm{S}-\mathrm{ABu})$ renforcés par des whiskers de cellulose, obtenus par évaporation de mélanges de suspensions aqueuses (système E).

Par contre, en dessous de $T g$, le renforcement est moins marqué. Par ailleurs, le niveau du module (Fig. 4b) au-dessus de $T g$ se maintient constant jusqu'à $500 \mathrm{~K}$ $\left(\approx 225^{\circ} \mathrm{C}\right)$, c'est-à-dire jusqu'à la dégradation de la cellulose (dans les mêmes conditions, la matrice seule serait devenue liquide, avant sa dégradation). Ces données ont été interprétées comme résultant de la formation d'un réseau tridimensionnel de whiskers de cellulose liés par des liaisons hydrogène se formant lors de l'évaporation de l'eau.

Lorsque la mise en ouvre passe par l'étape de lyophilisation (système XP), la formation de ces liaisons est beaucoup plus limitée. De plus, l'étape d'extrusion oriente localement les whiskers parallèlement les uns aux autres. De ce fait, l'effet de renfort, au-dessus de $T g$ de la matrice, est beaucoup moins élevé (cf. Fig. 6b).

En résumé, en dessous de $T g$ de la matrice, le renforcement dépend peu du procédé, alors qu'au-dessus de $\mathrm{Tg}$ (état caoutchoutique de la matrice polymère), cet effet en dépend fortement. On peut penser que cette dépendance est liée au type d'interactions entre particules renforçantes qui se développent lors de la mise en œuvre. L'importance des interactions entre particules sur le niveau de renforcement a pu être observé sur un grand nombre de systèmes (particules sphériques [4], «bâtons » [6], plaquettes [9]).

Pour évaluer le rôle du réseau percolant de charges ainsi que celui des interactions entre les renforts qui le constituent, il est nécessaire de confronter les résultats expérimentaux aux prédictions des différentes approches théoriques.

\section{Modélisation de systèmes composites}

La modélisation du comportement des matériaux composites peut faire appel à différents types d'approches, tels que les modèles micro-mécaniques ou des méthodes de type éléments discrets. La difficulté dans le cas des matériaux précédents est de déterminer un volume élémentaire représentatif du réseau de charges (au-delà du seuil de percolation) et de tenir compte de façon explicite des interactions entre charges ${ }^{2}$.

On peut d'abord comparer les résultats expérimentaux obtenus avec les composites à whiskers de cellulose avec le modèle analytique de Halpin-Kardos [10] (schématisé sur la Fig. 5a). Ce modèle de type champ moyen permet d'évaluer les modules de composites à fibres courtes en prenant en compte l'anisotropie des propriétés élastiques de ces fibres (module longitudinal des whiskers de l'ordre de $150 \mathrm{GPa}$, module transverse de l'ordre de $15 \mathrm{GPa}$ ). La figure 6 a indique clairement que les propriétés mesurées en dessous de $T g$ de la matrice sont proches du comportement prédit par ce modèle, ce qui n'est pas du tout le cas pour des températures supérieures à $T g$ (Fig. 6b). Pour prendre en compte l'effet de percolation des whiskers dans les matériaux « $\mathrm{E} »$, on peut utiliser un modèle « série-parallèle », où la fraction de phase rigide en parallèle correspond à la fraction de phase percolante (cf. Fig. 5), suivant une équation classique de percolation [11]. Bien que critiquable du point de vue de l'analyse des contraintes mécaniques dans les différentes phases, l'avantage d'un tel modèle, outre sa simplicité, est qu'il ne contient pas de paramètres ajustables. Une approche par éléments finis, faisant appel à un maillage particulier, a été utilisée par la suite et a confirmé les ordres de grandeurs obtenus [6]. On voit sur la figure $6 \mathrm{~b}$ que le modèle « de percolation » traduit bien le comportement des systèmes « $\mathrm{E} »$ (bien que, pour des fractions proches du seuil de percolation, ici de l'ordre de $1 \%$, un certain écart soit constaté), alors que le système « XP » est bien modélisé par l'équation d'Halpin-Kardos. Dans ce dernier cas, les whiskers ne forment pas de réseau, leur organisation locale empêchant leur percolation.

Il apparaît donc que lorsque la matrice est assez rigide, le réseau percolant contribue peu au renforcement mécanique, le transfert de contraintes matrice vers renfort

2 Programme Matériaux CNRS 1998-2000, « Mécanique des matériaux à microstructure bi-continue » A. Zaoui et J.Y. Cavaillé. 


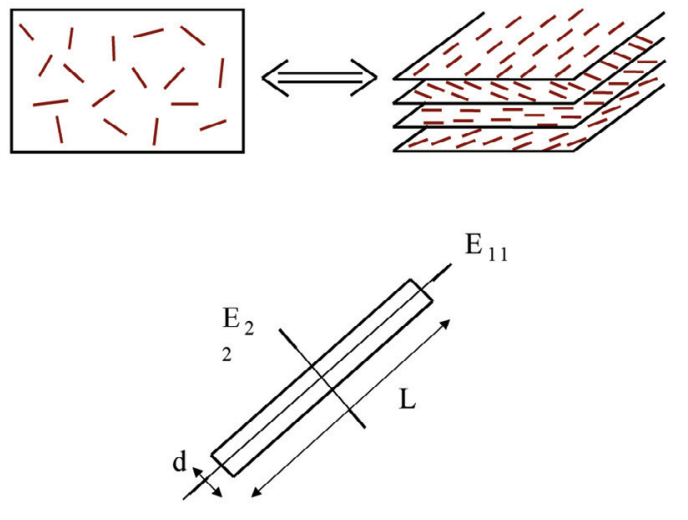

a
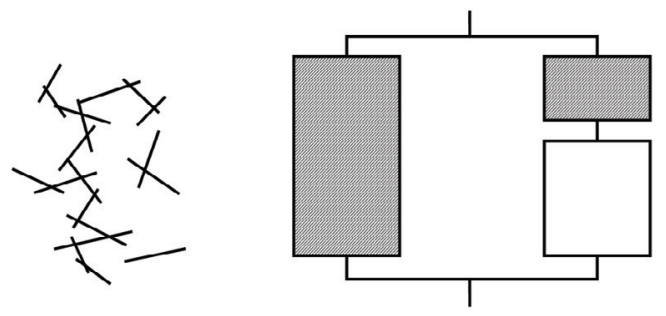

$$
\begin{aligned}
& \text { If } \mathrm{v}<\mathrm{v}_{\mathrm{c}} \text { then } \quad \psi=0 \\
& \text { If } \mathrm{v}>\mathrm{v}_{\mathrm{c}} \text { then } \quad \psi=\left\{\frac{\mathrm{v}-\mathrm{v}_{\mathrm{c}}}{1-\mathrm{v}_{\mathrm{c}}}\right\}^{\mathrm{b}}
\end{aligned}
$$

b

Fig. 5. À gauche, schématisation du modèle d'Halpin-Kardos. À droite, modèle série parallèle; la fraction de phase rigide percolante constitue la phase parallèle; $V_{c}$ est la fraction volumique au seuil de percolation, et $b$ l'exposant critique. Les valeurs de $V_{c}$ et $b$ sont déterminées numériquement ou par comparaison avec d'autres systèmes [7].

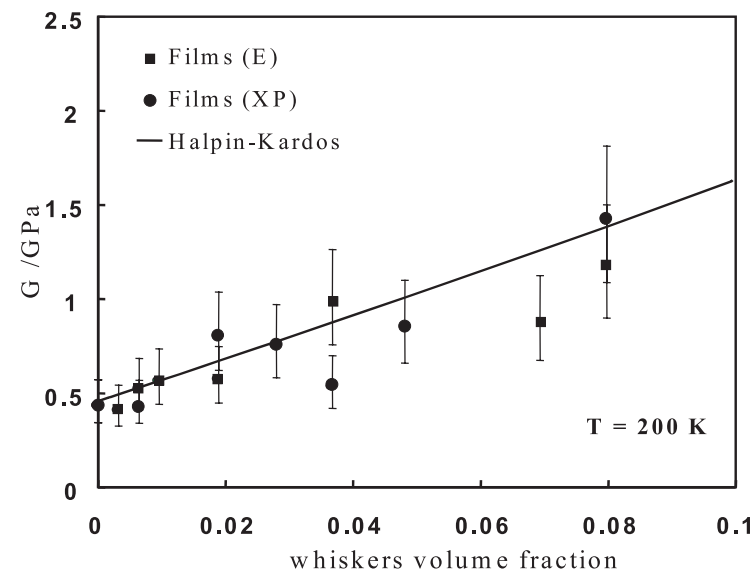

a

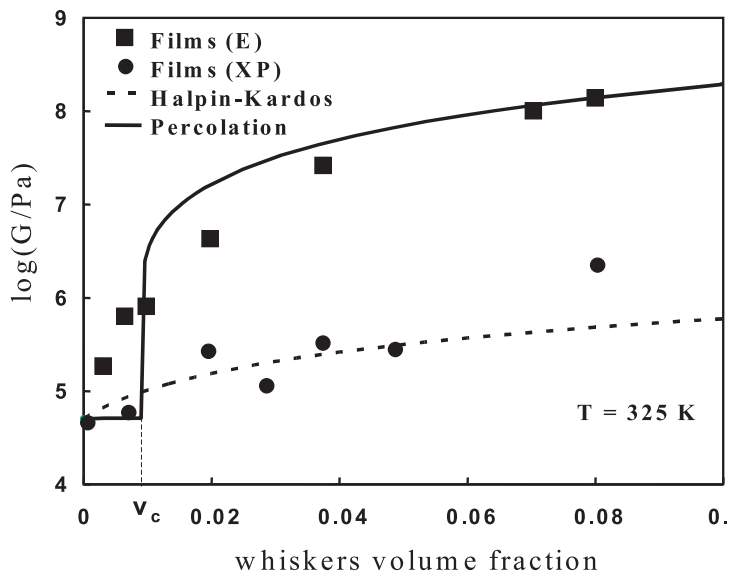

b

Fig. 6. À gauche, comparaison expériences et modèle d'Halpin Kardos pour différents procédés de mise en œuvre, lorsque la matrice est en dessous de $T g$. À droite, idem, mais au-dessus de $T g$.

jouant un rôle classique. Au contraire, pour une matrice souple, le réseau rigide intervient de façon prépondérante.

Cette analyse peut être étendue au cas des nanocomposites renforcés par des charges sphériques (système $\mathrm{PS} / \mathrm{PABu}$ décrit plus haut) pour lesquels on peut jouer sur la nature du contact entre sphères rigides. Là encore les approches par homogénéisation ou par éléments discrets ont été développées. Le modèle auto-cohérent généralisé à « $n$ phases » [12] a été utilisé pour modéliser les propriétés viscoélastiques de nanocomposites à matrice PABu renforcée par des particules sphériques de PS de diamètre $110 \mathrm{~nm}$ avant traitement thermique, c'est-àdire lorsque les particules de PS sont en contact mais n'ont pas coalescé. La description du système à l'aide du modèle 3-phases (faisant intervenir uniquement des inclusions sphériques PS dans une matrice PABu) sousestime les valeurs de renforcement au-dessus de $\mathrm{Tg}$ [5]. Dans le cadre de cette approche, il est nécessaire d'introduire des effets d'interphase (matrice « modifiée » en surface des particules dont la proportion augmente quand la taille de renfort diminue) par l'utilisation du motif à 4 phases représenté en figure 7a. Les propriétés de cette interphase, déterminées de manière inverse (c'est-àdire à partir du meilleur « fit » des courbes expérimentales par les courbes modélisées), peuvent ou non évoluer en fonction de la distance à la surface de la particule. Dans le cas des systèmes PS-PABu, l'épaisseur de l'interphase (13 nm) déduite par méthode inverse, s'avère être alors de l'ordre de grandeur du rayon de giration des chaînes macromoléculaires. On fait ici l'hypothèse forte de l'existence d'une interphase que les techniques expérimentales n'ont pas permis de mettre en évidence de manière indiscutable. Des mesures effectuées en RMN du solide l'accréditent, mais les mesures directes en calorimétrie ou en spectrométrie mécanique ou diélectrique ne permettent pas de trancher. Afin d'améliorer la description des résultats expérimentaux, il est peut-être préférable d'utiliser une autre approche, qui prend en compte explicitement les contacts entre particules.

Les méthodes d'éléments discrets permettent de prendre en compte explicitement ces contacts ainsi que la microstructure. De telles méthodes, initialement 


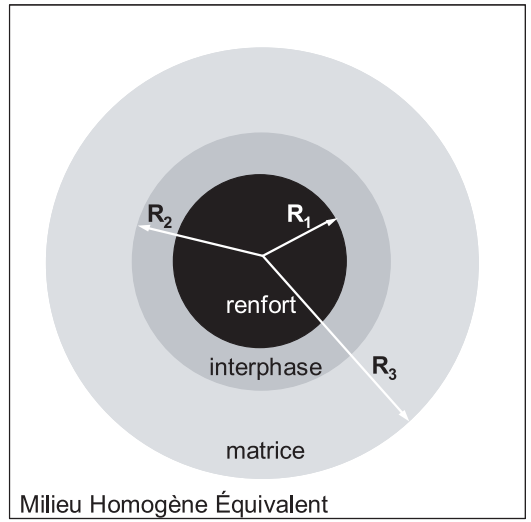

a

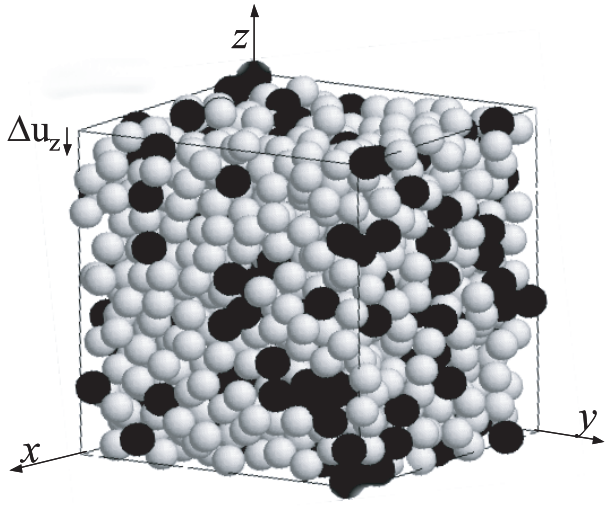

b

Fig. 7. À gauche, schéma d'un volume élémentaire représentatif à 4 phases utilisé dans une approche d'homogénéisation; à droite tirage au hasard dans une approche par éléments discrets.

développées en métallurgie des poudres [13], ont été appliquées à nos systèmes [14]. Les calculs sont réalisés sur un assemblage composite de particules sphériques (Fig. 7b) représentant soit le renfort rigide (sphères noires), soit la matrice souple (sphères blanches). À chaque contact entre particules voisines, trois forces et trois moments sont transmis. La «qualité »du contact, c'est-à-dire l'intensité de la transmission des efforts, est ajustée par l'intermédiaire d'un paramètre géométrique $\alpha=\left(R_{c} / R\right)^{2}$, où $R_{c}$ et $R$ désignent respectivement le rayon de contact et le rayon des particules : plus ce paramètre est grand $(0 \leq \alpha \leq 1)$, c'est-à-dire plus la surface de contact est grande, plus l'intensité de la transmission des moments est forte.

La figure 8a montre l'évolution du module normalisé de cisaillement des systèmes nanocomposites PS/PABu en fonction de la fraction volumique de PS, mesurée avant et après coalescence des particules voisines de PS : un seuil est clairement observé pour environ $20 \%$ de particules sphériques de PS après coalescence, alors que ce seuil n'apparaît pas avant coalescence. Ce module est calculé suivant l'expression :

$$
\text { Module normalisé }=1000 \cdot\left(\frac{G^{\prime}-G_{m}^{\prime}}{G_{r}^{\prime}-G_{m}^{\prime}}\right)
$$

où $G_{r}^{\prime}$ et $G_{m}^{\prime}$ sont les parties réelles des modules de cisaillement des phases rigides et molles, respectivement.

La figure $8 \mathrm{~b}$ montre des calculs effectués pour différents types d'interaction entre particules dures, en considérant un contraste entre phases dure et molle de $10^{12}$ (au lieu d'environ $10^{3}$ dans le cas précédent), afin d'exacerber les différences. Quand les interactions entre particules dures sont fortes (contact « liant» avec $\alpha=1$ ), le module effectif de l'assemblage augmente considérablement au seuil de percolation. Quand les interactions renfort/renfort sont faibles et le roulement des sphères dures est autorisé (contact « liant» avec $\alpha=10^{-6}$ ), il n'y a pas de discontinuité au seuil de percolation. Enfin, dans le cas d'un contact « glissant» ne résistant qu'aux efforts normaux, le module effectif augmente relativement peu avec la fraction volumique de renfort : l'assemblage se comporte comme un tas de sable sec. La question est alors de déterminer le type de liaisons pouvant intervenir dans le contact entre particules de PS, s'il est réalisé via des chaînes polymères de la matrice ou des liaisons type van der Waals. Dans le cas des composites après traitement thermique, le modèle décrit les résultats expérimentaux en supposant un contact rigide entre particules de PS. Par contre, dans le cas des systèmes filmifiés avant traitement, la description des résultats expérimentaux nécessite de prendre en compte une évolution de la rigidité des contacts avec la température [5]. On retrouve la notion d'interphase introduite précédemment dans le cas des modèles d'homogénéisation, mais cette fois son rôle est localisé au niveau des contacts particule-particule.

$\mathrm{Vu}$ l'importance des contacts entre particules dans le comportement des composites dans le domaine linéaire, on peut se poser la question du devenir, aux grandes déformations, des réseaux quelles forment.

\section{Cas des « grandes $»$ déformations (comportement non linéaire)}

Le comportement des matériaux composites sollicités aux grandes déformations (domaine non linéaire) dépend fortement de la température d'essai. En dessous de $T g$, par comparaison avec celui de la matrice, le comportement des composites se caractérise généralement par une contrainte d'écoulement élevée (dont l'augmentation est comparable à celle des modules) mais une rupture plus précoce. Il semble (mais il serait imprudent de généraliser) que la ténacité des matériaux nanocomposites (en fait l'allongement à la rupture) est supérieure à celle de leurs homologues à charges microniques [15]. Le problème de la modélisation vient du fait que les modèles de couplage mécanique sont pour la plupart dédiés au comportement élastique linéaire. Plusieurs méthodes de linéarisation sont envisageables, parmi lesquelles on peut citer les méthodes des sécantes ou des tangentes (Fig. 9). 


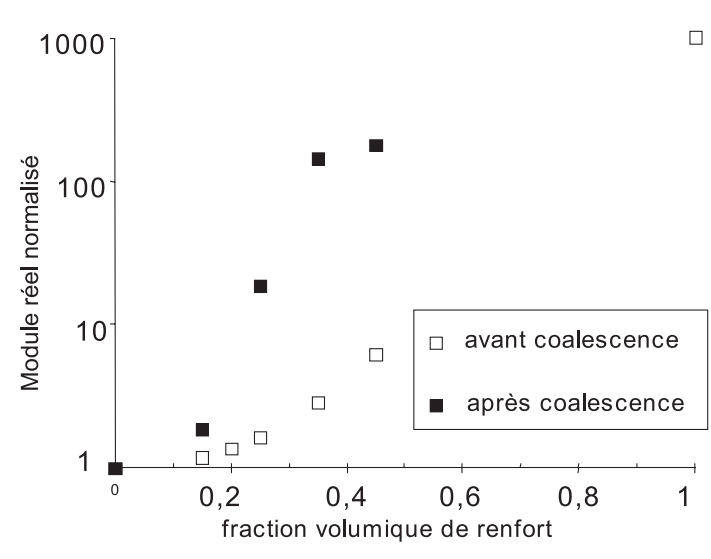

a
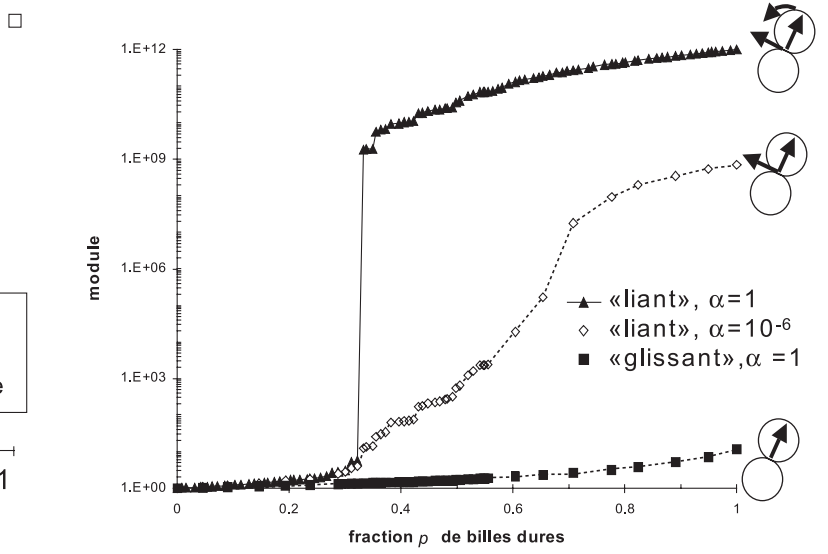

b

Fig. 8. (a) Comparaison des effets de renfort (échelle normalisée en module) de différents matériaux renforcés par des particules polymères rigides avant et après recuit. (b) Résultats de calcul par éléments discrets pour différents types d'interaction (même échelle que Fig. 8a, mais le contraste entre phases dures et molles $\left(10^{12}\right)$ est très exagéré pour exacerber les différences).

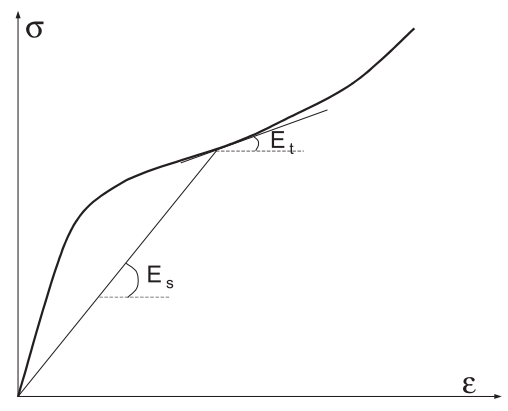

Fig. 9. Méthodes de linéarisation (module sécant ou module tangent). On passe de la matrice au composite en appliquant à chaque valeur du module de la matrice, le coefficient issu soit de l'expérience (en régime linéaire, essais isochrones ou isothermes), soit d'un modèle de couplage.

Plusieurs travaux [16] ont systématiquement montré que les courbes calculées surestiment le comportement réel. Parmi les raisons possibles, celle de l'apparition d'endommagement (décohésion, cavitation, etc.) doit être retenue.

Dans l'état caoutchoutique, la réponse mécanique des nanocomposites est très différente. L'introduction de particules de noir de carbone ou de silice dans les caoutchoucs est connue pour donner naissance à des effets non linéaires (effet Payne et effet Mullins). L'interprétation de ces effets est encore l'objet de controverse. Différents mécanismes sont avancés, soit sur la base de phénomènes d'adsorption des chaînes de la matrice sur les charges renforçantes, soit mettant en jeu l'existence d'un réseau de charges donc les contacts particule-particule $[2,17,18]$. On retrouve dans ces interprétations les concepts introduits précédemment dans la modélisation du domaine linéaire des nanocomposites au-dessus de leur température de transition vitreuse.

$\mathrm{Au}$ voisinage de la température de transition vitreuse, les nanocomposites à matrice $\mathrm{P}(\mathrm{S}-\mathrm{ABu})\left(\mathrm{Tg}=0{ }^{\circ} \mathrm{C}\right)$ renforcés par des particules de silice $(120 \mathrm{~nm})$ ont montré un comportement intermédiaire. Le domaine linéaire de l'essai de traction est correctement rendu par la modélisation

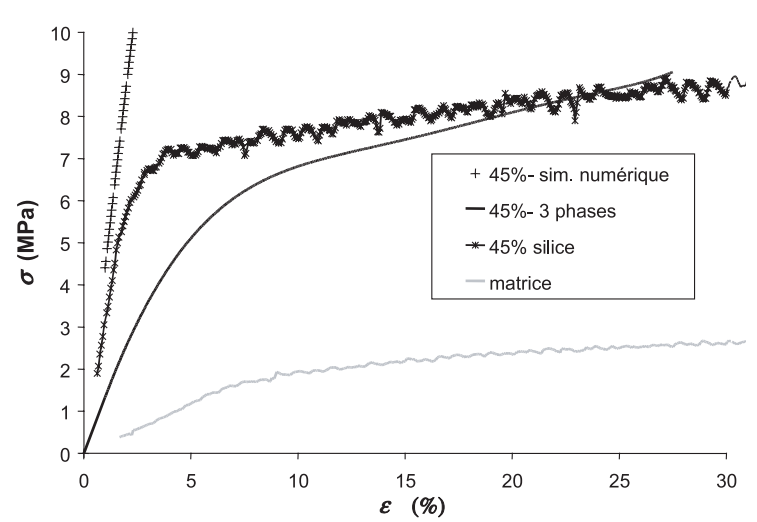

Fig. 10. Comportement en traction d'un nanocomposite (matrice $\mathrm{p}(\mathrm{S}-\mathrm{ABu})$ renforcé par $45 \%$ de silice $(T=0.98 \mathrm{Tg})$. Les simulations par éléments discrets et par homogénéisation encadrent les valeurs expérimentales [5].

par éléments discrets qui prend en compte les interactions entre charges de silice (liaisons « hydrogène »). Par contre, au-delà du seuil d'écoulement, la modélisation par homogénéisation (modèle 3-phases) s'avère plus réaliste. Il est probable que sous l'effet de l'effort de traction, le réseau percolant de silice se soit rompu et que le matériau soit simplement renforcé par transfert de contrainte de la matrice vers le renfort. Ce type de comportement (rupture du réseau de charge) a déjà été rencontré pour d'autres systèmes [19].

\section{Conclusions}

Comme pour tout matériau hétérogène, le comportement macroscopique des nanocomposites, dépend du comportement de chaque phase, de leur arrangement topologique, de la fraction volumique et des propriétés interfaciales. En fait, toutes les études confirment que l'interface devient de première importance quand les longueurs caractéristiques de la microstructure descendent 
jusqu'à l'échelle nanométrique. Au moins deux raisons peuvent être invoquées : d'abord la petite taille des charges conduit à une surface d'interface élevée ( $\sim 100 \mathrm{~m}^{2} \mathrm{~mm}^{-3}$ de matière), d'autre part dans le cas des matrices polymère, les distances entre charges deviennent comparables au rayon de giration des chaînes macromoléculaires. Cette dernière considération justifie le fait de porter une attention toute particulière aux nanocomposites à matrice polymère. Il est malheureusement difficile de comparer des matériaux ne se différenciant que par la taille des particules renforçantes, car les procédés de fabrication ajustent ces tailles en modifiant les caractéristiques interfaciales (tensioactifs, type de procédés, etc.). Dès lors, il est intéressant de comparer le comportement mécanique des nanocomposites à celui prédit par les approches de modélisation. Lorsque la matrice polymère est dans l'état vitreux, les modèles d'homogénéisation décrivent de manière satisfaisante le comportement linéaire des nanocomposites. Dans le domaine non linéaire, un effort reste à faire pour intégrer dans la description l'apparition de l'endommagement. Dans le cas des essais réalisés dans le domaine caoutchoutique, le comportement mécanique est fortement dépendant de l'existence ou non d'un réseau de charges percolant et de la nature des contacts qui le constituent. Si les approches intégrant les lois de percolation permettent de bien décrire le cas du réseau rigide, un effort reste à faire lorsque les interactions entre particules de renfort sont faibles, mais non négligeables [20]. La difficulté la plus grande est de relier les paramètres régissant le couplage particule/particule et particule/matrice aux caractéristiques physico-chimiques des phases constituant les matériaux. Une question souvent débattue et non réglée porte sur la nature de la couche interfaciale et la présence d'un gradient de mobilité. Bien évidemment, la difficulté pour décrire le comportement dans le domaine linéaire se rencontre aussi lorsque l'on s'intéresse au comportement au plus grandes déformations. En particulier, la question de l'endommagement des matériaux nanocomposites est complexe car ce dernier correspond à des tailles caractéristiques nanométriques elles aussi difficiles à observer.

Remerciements. Ces travaux ont pour partie été financés par le Programme Matériaux du CNRS (1998-2000), par un contrat Brite de la CE («Polymerizable and polymeric surfactants in emulsion for waterborne coatings ») et la société Elf-Atochem.

\section{Références}

[1] S.A. Wainwright, W.D. Biggs, J.D. Currey, J.M. Gosline, Mechanical design in organisms, Princeton Univ. Press, Princeton, NJ, 1976

[2] L. Chazeau, C. Gauthier, G. Vigier, J.Y. Cavaillé, Relationships between microstructural aspects and mechanical properties of polymer-based nanocomposites, in Handbook of organic-inorganic hybrid materials and nanocomposites, vol. 2; ed. H.S. Nalwa, American Scientific Publishers, 2003

[3] J.C. Daniel, Latex de particules structurées, Macromol. Chem. Symp. 10/11, (1985) 359

[4] L. Rios, M. Hidalgo, J.Y. Cavaillé, J. Guillot, A. Guyot, C. Pichot, Polystyrene poly(butyl acrylate-methacrylic acid) core shell emulsion polymers, Part I, Synthesis and colloidal characterization, Colloid Polym. Sci. 269 (1991) 812

[5] E. Chabert, Propriétés mécaniques de nanocomposites à matrice polymère : approche expérimentale et modélisation, Thèse Ph.D., INSA Lyon, 2002

[6] V. Favier, R. Dendievel, G.R. Canova, J.Y. Cavaillé, Acta Mater. 45 (1997) 1557-1565;

[7] V. Favier, Étude de nouveaux matériaux composites obtenus à partir de latex filmogènes et de whiskers de cellulose : effet de percolation mécanique, Thèse Ph.D., INPG, Lyon, 1994

[8] V. Favier, H. Chanzy, J.Y. Cavaillé, Polymer nanocomposites reinforced by cellulose whiskers, Macromolecules 28 (1995) 6365

[9] K. Varlot, E. Reynaud, M.H. Kloppfer, G. Vigier, J. Varlet, Clay-reinforced polyamide : prerencial orientation of the montmorill shits and the polya cristalline lamellae, J. Polym. Sci. Polym. Phys. 39 (2001) 1360

[10] J.C. Halpin, J.L. Kardos, Moduli of crystalline polymers employing composite theory, J. Appl. Phys. 43 (1972) 2235

[11] N. Ouali, J.Y Cavaillé, J. Perez, Elastic, Viscoelastic and Plastic behavior of multiphase polymer blends, Plast. Rubber and Composites Proc. Appl. 16 (1991) 55

[12] E. Hervé, A. Zaoui, A N-layered inclusion-based micromechanical modelling, Int. J. Eng. Sci. 31 (1993) 1

[13] A. Jagota, G. Scherrer, Viscosities and sintering rates of composites packing of spheres, J. Am. Ceram. Soc. 78 (1995) 521

[14] E. Chabert, R. Dendievel, C. Gauthier, J.-Y. Cavaillé, Prediction of the elastic response of polymer based nanocomposites : a mean field approach and a discrete simulation, submitted to Compos. Sci. Technol.

[15] M. Sumita, T. Shimuza, K. Miyasaka, K. Ishikawa, Effect of reducible properties of temperature, rate of strain, and filler content on the tensile yield stress of nylon 6 composites filled with ultrafine particles, J. Macromol. Sci. Phys. 22 (1983) 601

[16] L. Chazeau, Étude de nanocomposites à renfort cellulosique et matrice PVC : mise en œuvre, étude structurale, comportement mécanique, Thèse Ph.D. UJF, Grenoble, 1998 ; L. Chazeau, J. Perez, J.Y. Cavaillé, J. Polym. Sci. Polym. Phys. 38 (2000) 383-392

[17] L. Chazeau, J.D. Brown, L.C. Yanyo, S.S. Sternstein, Modulus recovery kintics and other insights into the Payne effect for filled elastomers, Polym. Comp. 21 (2000) 202

[18] C. Gauthier, Diplôme d'Habilitation à Diriger des Recherches (DHDR), INSA, Lyon, 2001

[19] P. Hajji, J.Y. Cavaillé, V. Favier, C. Gauthier, G. Vigier, Tensile behavior of new nanocomposite materials from latex and cellulose whiskers, Polymer Composites 17 (1996) 612

[20] E. Chabert, M. Bornert, C. Gauthier, J.Y. Cavaillé, A. Zaoui, Mechanical properties of co-continuous structured nanocomposite materials, soumise à Mat. Sci. Eng. 